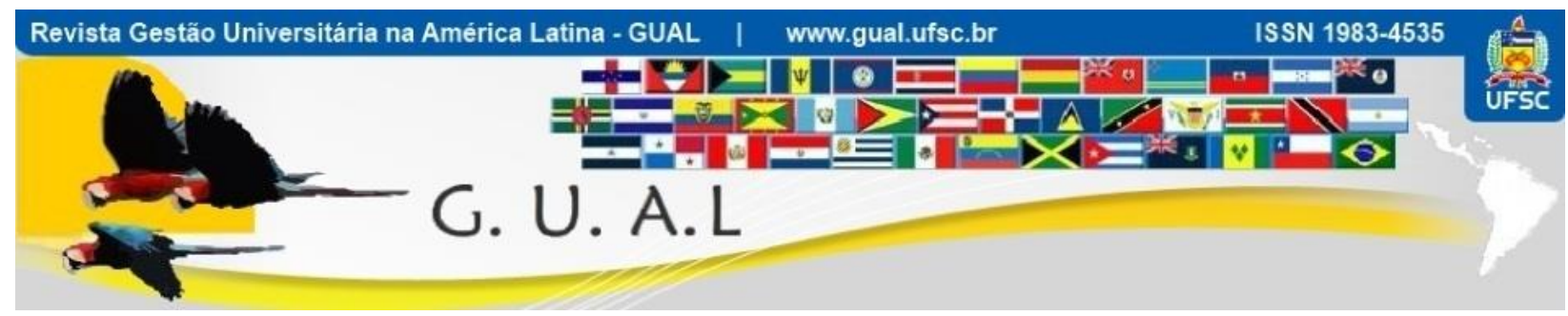

DOI: http://dx.doi.org/10.5007/1983-4535.2014v7n3p247

\title{
A INFLUÊNCIA DA CULTURA ORGANIZACIONAL UNIVERSITÁRIA SOBRE O PROCESSO DE TRANSFERÊNCIA TECNOLÓGICA
}

\section{THE INFLUENCE OF THE ORGANIZATIONAL CULTURE ON UNIVERSITY TECHNOLOGY TRANSFER PROCESS}

Dusan Schreiber, Doutor

Universidade Feevale dusan@feevale.br

Recebido em 21/janeiro/2013

Aprovado em 23/julho/2014

Sistema de Avaliação: Double Blind Review

Esta obra está sob uma Licença Creative Commons Atribuição-Uso. 


\title{
A INFLUÊNCIA DA CULTURA ORGANIZACIONAL UNIVERSITÁRIA SOBRE O PROCESSO DE

\section{RESUMO}

O atual ritmo do desenvolvimento tecnológico obriga as empresas não apenas manter investimentos em inovação como aumentar continuamente o valor aplicado devido ao crescente custo de P\&D. As instituições de ensino, por sua vez, detêm o conhecimento técnico, pessoal especializado e infra-estrutura laboratorial de alto nível e apresentam interesse em se aproximar do segmento empresarial, visando aplicar os conhecimentos gerados dentro dos seus centros de pesquisa. Os governos, com o objetivo de fazer frente à globalização e garantir a sustentabilidade econômica das empresas nacionais, procuram, de várias formas, criar mecanismos para fomentar a consolidação do parque fabril local, com a geração de empregos e de renda. Desta convergência de motivos, finalidades e necessidades dos três atores, das empresas, das instituições de ensino e dos governos, surgiu a configuração de um novo modelo de desenvolvimento tecnológico denominado de "Hélice Tripla", onde os governos criam fundos com recursos financeiros que são liberados para o desenvolvimento de projetos e de inovações, com a condição sine qua non de que os mesmos sejam aplicados em projetos executados em parceria entre as instituições de ensino/ pesquisa e as empresas. A presente pesquisa tem por objetivo avaliar o grau de influência da cultura organizacional de um grupo específico de profissionais, constituído por pesquisadores e docentes de seis cursos tecnológicos de uma grande universidade privada do sul do Brasil, no processo de transferência tecnológica.

Palavras-chave: Hélice-Tripla. Cultura Organizacional. Transferência Tecnológica.

\begin{abstract}
The current trend of technological development not only requires companies to maintain investment in innovation as to continuously increase the value of investment for $R \& D$. Educational institutions, in turn, hold the expertise, trained personnel and laboratory infrastructure and interest in being closer to the business segment, in order to apply the knowledge generated within their research centers. Governments, interested to provide economic sustainability for national companies, attempt in various ways to create mechanisms to foster consolidation of local manufacturing facilities, with the creation of jobs and income. This convergence of motives, purposes and needs of the three economic actors, businesses, educational and government institutions, originated a new model of technological development called the " Triple Helix ", with governments creating funds with funds for development of the projects and innovation, with the prerequisite that they be invested in projects in partnership between educational institutions / research and business. This research aims to assess the degree of influence of the organizational culture of a specific group of professionals, consisting of researchers and teachers from six technology courses at a large private university in southern Brazil, in the technology transfer process.
\end{abstract}

Keywords: Triple-helix. Organizational Culture. Technology Transfer. 


\section{A INFLUÊNCIA DA CULTURA ORGANIZACIONAL UNIVERSITÁRIA SOBRE O PROCESSO DE

\section{INTRODUÇÃO}

A aceleração do ritmo de desenvolvimento tecnológico obriga as empresas a aumentar continuamente o valor aplicado em $\mathrm{P} \& \mathrm{D}$, devido ao custo de profissionais, dos equipamentos e da taxa de inovações não viáveis comercialmente. Por outro lado as instituições de ensino procuram interagir com as empresas, visando aplicar os conhecimentos gerados dentro de seus centros de pesquisa. Os governos, dentro do seu papel de garantir a sustentabilidade econômica das empresas nacionais, com a geração de empregos e de renda, também percebem a necessidade de criar mecanismos para fomentar a inovação tecnológica nacional.

Desta convergência dos motivos, finalidades e necessidades dos três atores emerge um modelo de desenvolvimento tecnológico onde o governo libera recursos financeiros para projetos e inovações dentro da interação universidade-empresa. Apesar deste incentivo o que se verifica na realidade brasileira é uma taxa de desenvolvimentos conjuntos aquém do esperado e não condizente com o volume de recursos disponibilizados pelo governo brasileiro para este fim. Entre as causas constatadas destaca-se a diferença entre a cultura organizacional das instituições de ensino e as empresas.

A cultura organizacional é definida a partir de pesquisas mais recentes como uma amálgama de culturas de grupos menores tais como áreas, departamentos, unidades de negócios corporativos e que influenciam as rotinas e práticas organizacionais. A presente pesquisa tem por objetivo avaliar o grau de influência da cultura organizacional em um grupo específico de profissionais, sobre o processo de transferência tecnológica.

A pesquisa foi conduzida utilizando o método de estudo de caso, por ser indicado para uma análise intensiva de uma situação particular e preferencialmente para estudar eventos contemporâneos e também por se entender que apresenta melhor aderência ao objetivo e às questões que nortearam o estudo. Nesse trabalho procurou-se realizar uma análise descritiva, a partir de entrevistas com vinte e quatro profissionais, entre gestores universitários, coordenadores e professores de seis cursos tecnológicos de uma grande universidade privada do sul do Brasil.

A partir dos resultados obtidos foi possível identificar idiossincrasias de culturas organizacionais de grupos específicos, como é o caso de grupo de docentes e pesquisadores dos cursos pesquisados, que mantêm o seu próprio conjunto de valores, regras, crenças e percepções distantes do discurso institucional da universidade, contribuindo para o baixo grau de interação universidade-empresa para a transferência tecnológica. 


\section{A INFLUÊNCIA DA CULTURA ORGANIZACIONAL UNIVERSITÁRIA SOBRE O PROCESSO DE

\section{INTERAÇÃO UNIVERSIDADE-EMPRESA}

Com base em resultados de sua pesquisa sobre os resultados da interação U-E na Unicamp, Brisolla et al. (1997) afirmou que apesar da convicção generalizada de que as universidades devem e podem desempenhar um papel muito importante no encurtamento do gap tecnológico que constitui sério obstáculo para o crescimento econômico do País ainda não há consenso sobre a melhor forma de interação. A autora entende que as tentativas de o governo de incentivar o estreitamento de laços entre universidade e empresa obtiveram resultados aquém do esperado. No entanto, percebe-se que o ambiente de competição intensa no mercado vem obrigando as empresas a buscar parceria com instituições de ensino e pesquisa. Além disso, identificam-se novas configurações para os sistemas de inovação redes de inter-relação entre empresas, universidades e institutos de pesquisa. Nessas redes, que constituem a base da nova sociedade do conhecimento, a autora entende que a universidade terá necessariamente um papel relevante.

Em estudo realizado sobre o desenvolvimento da América Latina, Sabato e Botana (1975) destacaram que a inovação encontra diversos obstáculos e enfrentá-los exige do governo, da estrutura produtiva e da infra-estrutura científico-tecnológica. A atuação do governo tem como objetivo formular e implementar políticas no âmbito científico-tecnológico de forma deliberada; a infra-estrutura científico-tecnológica é responsável pela capacidade criadora, desenvolvendo a ciência e a tecnologia, e a estrutura produtiva tem como função revolucionar o sistema de produção, através de inovações. O relacionamento entre estrutura produtiva e instituições de ensino e pesquisa é a mais difícil.

Outros autores conceberam modelos semelhantes. O principal deles é a "Hélice Tripla” de Leydesdorff e Etzkowitz (1996). O modelo toma as formas tradicionais de diferenciação institucional entre as universidades, empresas e governo, como o ponto de partida. A perspectiva evolucionária agrega a esta configuração histórica a noção de que as pessoas modelam, de forma reflexiva, estas instituições. O referido modelo leva em conta o crescente papel do setor de conhecimento em relação à infra-estrutura econômica e política da sociedade. A diferença entre nação estado e economia leva à análise de diferenciação funcional entre a ciência e mercado e de diferenciação institucional entre o controle privado e público. Percebem-se, desta forma, três dinâmicas claras e distintas: (a) a dinâmica econômica do mercado; (b) a dinâmica interna da produção do conhecimento e (c) a governança da interface em diferentes níveis. 


\section{A INFLUÊNCIA DA CULTURA ORGANIZACIONAL UNIVERSITÁRIA SOBRE O PROCESSO DE

Um importante contraponto ao modelo de "Hélice Tripla" foi apresentado por Tuunaine (2002). Os resultados de pesquisa empírica conduzida pelo autor evidenciam que o modelo necessita de estudos mais aprofundados e debates nos círculos científicos. O autor destaca que (i) o modelo precisa de maior foco quando se tratar de distinção analítica entre as dimensões teórica, metodológica e aplicada do programa de pesquisa local; (ii) o modelo não oferece subsídios satisfatórios em relação a apropriação dos resultados comerciais da pesquisa, dentro da interação U-E, principalmente na questão de propriedade de patentes da pesquisa, na solução dos problemas na transferência do conhecimento da academia para o mercado e na orientação de como coordenar as relações entre o grupo de pesquisadores e a empresa constituída para aplicar seus resultados com fins comerciais.

Leydesdorff e Etzkowitz (2002) destacam como crítica que o modelo não pode ser reificado e nem pode substituir os debates concernentes às abordagens conceituais e empíricas de desenvolvimento de cada um dos atores, além de contestar a vertente crítica que defende a inclusão da sociedade civil como mais um ator na Hélice, por considerar que a mesma já está sendo representada pelo governo. Uma sugestão é que a adaptação do modelo às necessidades de desenvolvimento econômico e social local e regional é pertinente e viável.

Terra e Etzkowitz (2007) destacam a importância da capacidade de adaptação dos três atores que compõem a "Hélice Trípla", às contingências ambientais, em permanente transformação. Neste contexto a empresa, de base tecnológica, deverá estar ancorada na fabricação de produtos inovadores, além de estar interagindo com as universidades e centros de pesquisa. A universidade, por sua vez, deverá possuir o perfil de universidade empreendedora, com tarefa de promover o desenvolvimento econômico e social, através de novas estruturas organizacionais e com a missão de garantir a inserção no mercado globalizado, de todo o seu corpo docente e discente. E, finalmente, o governo, denominado por Terra e Etzkowitz (2007) como "governo da nova era", deverá ser participativo, viabilizando uma interação efetiva com os demais atores, dialogando e construindo com base no consenso o plano político que possibilite a efetiva parceria.

As universidades estão, atualmente, passando pela fase de "Segunda Revolução", ao incorporar o desenvolvimento econômico e social como parte de sua missão (ETZKOWITZ, 1998). Esta revolução agrega novos significados à "Primeira Revolução" que compreendeu a inclusão da função de pesquisa na missão das instituições de ensino, que, até então, estavam voltadas apenas à atividade de ensino. Apesar de reconhecer que muitas universidades ainda 


\section{A INFLUÊNCIA DA CULTURA ORGANIZACIONAL UNIVERSITÁRIA SOBRE O PROCESSO DE TRANSFERÉNCIA TECNOLÓGICA \\ DOI: http://dx.doi.org/10.5007/1983-4535.2014v7n3p247}

não completaram sequer esta etapa, observa-se que as universidades que queiram desempenhar o seu papel de agente ativo na sociedade, devem aderir à "Segunda Revolução".

Gunasekara (2006) propõe um modelo conceitual para analisar a variação nos papéis desempenhada por universidades no desenvolvimento dos sistemas regionais de inovação. Este modelo consiste de duas partes que exploram "o quê" as universidade fazem e "por quê", correlacionando com o modelo original de "Hélice Tripla", revisado com base em literatura sobre o engajamento das universidades em atividades de inovação. $\mathrm{O}$ autor testou o modelo empiricamente em três universidades e estudo evidenciou que as universidades possuem reduzida competência empreendedora na comercialização dos resultados de suas pesquisas no mercado, mas que, no entanto, o seu envolvimento em ações de desenvolvimento regional é significativo.

Oughton, Landabaso e Morgan (2002) estudam o paradoxo de inovação regional e suas implicações. O referido paradoxo se refere à aparente contradição entre a maior necessidade de investimento para atividades inovativas em regiões menos desenvolvidas e a relativamente reduzida capacidade destas regiões de absorver os fundos públicos destinados à promoção de inovação em comparação a investimentos realizados em regiões mais desenvolvidas. As análises empíricas da natureza do paradoxo evidenciam forte complementaridade entre negócios, pesquisa e investimento governamental em P\&D, mas demonstram que as políticas de inovação e tecnologia e políticas de fomento industrial não estão alinhadas.

Os resultados das pesquisas mais recentes apontam para a necessidade de maior compreensão da dimensão cultural dos agentes envolvidos no processo e destacam a importância dos fatores comportamentais e cognitivos para o sucesso da efetiva interação. $\mathrm{Na}$ seção a seguir são apresentados os conceitos mais relevantes da cultura organizacional.

\section{CULTURA ORGANIZACIONAL}

Para Souza (1978) a cultura pode ser dividida em três elementos, cada um abrangendo uma série de fenômenos, interdependentes e não necessariamente equivalentes. São eles: (i) preceitos compreendidos como conjunto de normas, valores, regulamentos, política administrativa, tradições, estilos gerenciais, questões formais (leis), assim como costumes, rituais, padrões e aspectos informais que são seguidos tacitamente; (ii) tecnologia, sendo entendida como conjunto de instrumentos, processos, layout, distribuição de tarefas, divisão 


\section{A INFLUÊNCIA DA CULTURA ORGANIZACIONAL UNIVERSITÁRIA SOBRE O PROCESSO DE TRANSFERÉNCIA TECNOLÓGICA \\ DOI: http://dx.doi.org/10.5007/1983-4535.2014v7n3p247}

de trabalho e fluxo organizacional subsidiando a função técnica, metodológica científica, racional e operativa da organização; (iii) caráter como um conjunto de expressões ativas e afetivas dos indivíduos da organização, manifestações subjetivas, as percepções, os sentimentos e as reações positivas ou negativas dos sujeitos organizacionais características dos comportamentos grupais.

A cultura organizacional é um conjunto de pressupostos básicos que um grupo inventou, descobriu ou desenvolveu ao aprender como lidar com os problemas de adaptação externa e integração interna. Estes pressupostos funcionaram bem o suficiente para serem considerados válidos e ensinados a novos membros como forma correta de perceber, pensar e sentir em relação a esses problemas. A aprendizagem cultural se realiza em vários níveis: (i) nível dos artefatos visíveis, que são de fácil percepção, mas de difícil interpretação, como, por exemplo o comportamento das pessoas, o layout da empresa, os documentos; (ii) nível dos valores que governam o comportamento das pessoas onde existem diferenças entre os valores aparentes e os valores de uso; (iii) nível dos pressupostos básicos, que são geralmente inconscientes, mas demonstram como os membros de um grupo percebem, pensam e sentem (SCHEIN,1988).

Não só a cultura é diferente de organização para organização, em virtude dos conteúdos preceituais e de características específicas, como dentro da mesma organização formam-se subculturas diferentes nas diversas unidades (divisões, departamentos, seções). Se um grupo compartilha durante algum tempo um número de experiências, lidando com problemas internos e externos, pode-se concluir que o grupo irá compartilhar uma visão de mundo (SOUZA, 1978). A autora constata que freqüentemente os grupos com background ocupacional semelhante tendem a desenvolver culturas próprias no interior das organizações. Pessoas em associação contínua desenvolvem traços comportamentais e mecanismos culturais que são únicos àquele grupo e diferem de alguma forma dos de outros grupos e de complexo sócio-cultural mais amplo. Todo o grupo caracterizado pela interação contínua desenvolve variações culturais e um corpo de relações sociais peculiares e próprios aos seus membros, sendo que os grupos são os blocos de construção de uma organização. Em organizações de maior porte, torna-se impossível a interação contínua entre todos os seus membros.

A cultura, para Motta e Caldas (1997), é um conceito antropológico e sociológico que oportuniza várias definições: (i) a forma pela qual uma comunidade satisfaz a suas necessidades materiais e psicossociais, sendo implícita a noção de ambiente como fonte de 


\section{A INFLUÊNCIA DA CULTURA ORGANIZACIONAL UNIVERSITÁRIA SOBRE O PROCESSO DE

sobrevivência e crescimento, (ii) a adaptação em si, ou seja, a forma pela qual uma comunidade define seu perfil em função da necessidade de adaptação ao meio ambiente. Autores evidenciam que as empresas brasileiras são marcadas por decisões que tendem a ser caracterizadas por uma interação social intensa, bem como por um envolvimento ativo dos dirigentes superiores, geralmente autocráticos. Essa interação não se restringe somente ao mesmo nível hierárquico, mas encontra-se entre os diferentes níveis nas organizações.

A cultura implica estabilidade, enfatiza demonstrações conceituais, serve como fator aglutinador para levar os membros do grupo em direção ao consenso, implica dinâmica e padronização. A cultura, com a construção do significado social e normativo possibilita que um grupo se fortaleça ou se desintegre, exerce pressão sobre os membros da organização para compeli-los a obedecer ao código compartilhado e molda os comportamentos individuais. A cultura expressa os valores e as crenças que os membros desse grupo partilham. Tais valores manifestam-se por meio de símbolos, como mitos, rituais, histórias, lendas e uma linguagem especializada, orientando os indivíduos de uma referida cultura na forma de pensar, agir e tomar decisões. Pesquisas identificaram os atributos culturais, tais como a liberdade de compartilhamento de informações, o trabalho em equipe, confiança, respeito e entusiasmo (PIRES e MACEDO, 2006; SUGATO, 1994).

Segundo Bolon e Bolon (1994), a maioria das organizações não podem ser consideradas idioculturas, que são os traços culturais idiossincráticos que emergem quando um grupo de pessoas em efetiva interação identifica um problema comum para todos os membros do grupo. Os autores destacam que a formação de idioculturas é também reforçada por certas características estruturais. A especialização e a divisão de trabalho permitem à organização concentrar a expertise e a tecnologia em certas áreas. Ao mesmo tempo em que esta forma de gestão organizacional possibilita maior eficiência ela também gera diferenças entre grupos nas suas percepções do ambiente. Como cada grupo desenvolve suas próprias crenças, valores, constatações, fontes de informações e linguagem, entende-se que o tecido organizacional é propício à proliferação idiocultural (BOLON e BOLON, 1994).

A identidade de uma organização, definida a partir de representações construídas por seus atores organizacionais, orienta a ação destes no contexto do seu trabalho nas empresas. Verifica-se que é possível compreender a identidade de uma organização por meio de traços da cultura, e que os elementos culturais fornecem uma compreensão das representações da organização, revelando não o que é comum a todas as organizações, mas o que é peculiar e 


\section{A INFLUÊNCIA DA CULTURA ORGANIZACIONAL UNIVERSITÁRIA SOBRE O PROCESSO DE TRANSFERÉNCIA TECNOLÓGICA \\ DOI: http://dx.doi.org/10.5007/1983-4535.2014v7n3p247}

particular. A compreensão da identidade é enriquecida quando se analisam as narrativas a partir de traços culturais, sendo que esses dão significado à identidade, ou seja, não se pode compreender a identidade sem considerar a cultura e vice-versa (MACHADO, 2005).

Para Magee (2002) a cultura organizacional é composta por suposições explícitas e tácitas ou entendimentos em comum de um grupo de pessoas; uma configuração particular de suposições e compreensões é distinta a um determinado grupo; estas suposições e entendimentos servem como critérios de aceite ou rejeição de percepções, pensamentos, sentimentos e comportamentos; eles são aprendidos e passados para novos membros de um grupo através de interação social; e cultura é dinâmica - ela muda ao longo do tempo, apesar de que as suposições tácitas ligadas ao cerne da cultura organizacional são mais resistentes à mudança. Reconhece quatro tipos de conhecimento cultural: conceitual, descritivo, prescritivo e axiomático.

Com base nos resultados da pesquisa para tese de doutorado Jerry Plymire (1993) evidenciou que a cultura organizacional não é representada por uma cultura única que permeia a organização como um todo e sim, é caracterizada por aspectos e traços culturais distintos cuja intensidade varia de departamento a departamento. Desta forma o autor percebe a cultura organizacional como multidimensional, ou seja, uma amálgama de características idiossincráticas e não apenas um composto de duas ou mais características.

Berthon, Pitt e Ewing (2001) verificaram que à medida que as organizações se tornam maduras e crescem, o seu repertório de esquemas cognitivos se torna mais desenvolvido e as organizações se tornam mais passivas na busca de informações. Os autores identificaram que a cultura organizacional exerce influência sobre o processo decisório gerencial em termos de modelos mentais dos gestores organizacionais, no tocante a percepção de oportunidades, riscos, baseando-se em memória organizacional.

Hersey, Blanchard e Johnson (1996) afirmam que a cultura influencia como as decisões são tomadas, o estilo de gestão e relações e padrões comportamentais da organização, em que confirmam a percepção de Chin-Loy e Huizenga (2003) para quem um grande número de falhas organizacionais pode resultar de falta de alinhamento da cultura organizacional e estratégia. Na percepção de Sugato (1994) a organização deverá avaliar e compreender os atributos culturais antes de promover qualquer tipo de ajuste organizacional. 


\section{A INFLUÊNCIA DA CULTURA ORGANIZACIONAL UNIVERSITÁRIA SOBRE O PROCESSO DE

\section{MÉTODO}

O método de pesquisa escolhido para a pesquisa foi o estudo de caso, por se entender que apresenta melhor aderência ao objetivo e às questões que nortearam o estudo. Tull (1976, p 323) afirma que "um estudo de caso refere-se a uma análise intensiva de uma situação particular". De acordo com Yin (1989), a preferência pelo uso do Estudo de Caso deve ser quando do estudo de eventos contemporâneos, em situações onde os comportamentos relevantes não podem ser manipulados, mas onde é possível se fazer observações diretas e entrevistas sistemáticas.

O estudo de caso é útil, segundo Bonoma (1985, p. 207), "... quando um fenômeno é amplo e complexo, onde o corpo de conhecimentos existente é insuficiente para permitir a proposição de questões causais e quando um fenômeno não pode ser estudado fora do contexto no qual ele naturalmente ocorre". Os objetivos do Método do Estudo de Caso não são a quantificação ou a enumeração, "... mas, ao invés disto (1) descrição, (2) classificação (desenvolvimento de tipologia), (3) desenvolvimento teórico e (4) o teste limitado da teoria. Em uma palavra, o objetivo é compreensão" (p. 206). Dentre as aplicações para o estudo de caso citado por Yin (1989), nesse trabalho procurou-se descrever o contexto da vida real e realizar uma avaliação descritiva.

O estudo de caso foi realizado em uma universidade privada, de grande porte, localizada na região metropolitana de Porto Alegre e com quase quarenta anos de existência. Atualmente a instituição oferece mais de cinqüenta cursos de graduação e vinte cursos de pósgraduação em nível de mestrado e doutorado. O seu quadro de colaboradores é composto por aproximadamente 1.800 pessoas, sendo metade do corpo docente e outra metade de funcionários de apoio e gestão. Durante as três primeiras décadas de sua existência primou apenas pela excelência do ensino, sendo forçada, a partir da década de noventa a rever o seu foco e incluir novos objetivos institucionais, tais como desenvolvimento setorial e regional, maior interatividade com a comunidade local e regional, além de inclusão de programas sociais.

A presente pesquisa foi realizada com o objetivo de procurar identificar e dimensionar as variáveis que tiveram maior influência sobre o processo de aproximação do mercado. O estudo foi conduzido no período de Março a Julho de 2012, por meio de realização de vinte e quatro entrevistas individuais em profundidade. $\mathrm{O}$ universo selecionado foi composto por 


\section{A INFLUÊNCIA DA CULTURA ORGANIZACIONAL UNIVERSITÁRIA SOBRE O PROCESSO DE TRANSFERÉNCIA TECNOLÓGICA \\ DOI: http://dx.doi.org/10.5007/1983-4535.2014v7n3p247}

doze professores universitários, seis coordenadores, dos cursos tecnológicos e seis gestores universitários responsáveis pela tarefa de conduzir o processo de interação U-E.

Os cursos tecnológicos selecionados com base na sua adequação aos objetivos da pesquisa, eram os de Engenharia Mecânica, Engenharia de Produção, Engenharia Civil, Engenharia de Alimentos, Engenharia Elétrica, Arquitetura e Urbanismo. A referida aderência aos objetivos da pesquisa baseia-se no critério central do estudo, que é o vetor tecnológico associado à transferência do conhecimento científico com fins de sua aplicação em ambiente produtivo. A seleção dos professores foi decorrente da indicação dos coordenadores dos respectivos cursos de graduação.

Todas as entrevistas foram realizadas em salas de reuniões na própria universidade, gravadas em áudio, em períodos temporais que variavam de 60 a 100 minutos. A seqüência de entrevistas foi iniciada pelos coordenadores dos seis cursos tecnológicos, seguida pelos docentes e finalizada pelas entrevistas com os gestores administrativos das unidades de pesquisa e pós-graduação e de educação continuada.

$\mathrm{Na}$ etapa seguinte as gravações foram transcritas, viabilizando a terceira etapa de tratamento, de análise de conteúdo, codificação axial e reconstrução das narrativas, por meio de agrupação dos dados, sua contagem, comparação e aplicação de analogias. Os dados foram contrastados, com o objetivo de separar as variáveis, vinculando as especificidades às categorias genéricas, substituindo as variáveis por fatores, consistindo relações entre as variáveis, construindo a cadeia lógica de evidências, criando a coerência teórica.

\section{ANÁLISE DE RESULTADOS}

As condições do mercado, com o surgimento de novas instituições de ensino procurando se estabelecer e assegurar o seu espaço, exigiram da instituição pesquisada a mudança no foco estratégico institucional - excelência do ensino. Obrigada a dividir o mercado de ensino, até então cativo, a universidade reviu a sua estratégia de atuação e elaborou um projeto de mudanças, visando adequar a sua estrutura interna às novas condições e assim permitir a sua viabilidade econômica e financeira (TERRA \& ETZKOWITZ, 2007). O conjunto de alterações estruturais impactaria diretamente no "modus vivendi" do seu corpo funcional, até então estável e protegido do ambiente externo, que assumiu características de competitividade e agressividade mercadológica (MAGEE, 2002). 


\section{A INFLUÊNCIA DA CULTURA ORGANIZACIONAL UNIVERSITÁRIA SOBRE O PROCESSO DE

Diferentemente do que foi previsto nos planos de ação institucionais, várias das alterações planejadas e implementadas não obtiveram os resultados desejados. Um dos programas onde foi identificado o maior distanciamento entre a previsão e a realização foi o de interação da universidade com as empresas, com o foco na prestação de serviços em pesquisa e desenvolvimento, inovações incrementais e radicais em produtos e processos. Apesar de investimentos realizados, pela universidade, em infra-estrutura, como laboratórios e criação de departamento dedicado à interação com o mercado, os resultados obtidos ficaram aquém do esperado (SABATO \& BOTANA, 1975; LEYDESDORFF \& ETZKOWITZ, 2007).

Alinhado com a nova estratégia da universidade voltada ao desenvolvimento regional e setorial, trabalho comunitário e programas de inclusão social, a fim de se legitimar perante a sociedade e as demais instituições de ensino concorrentes, além de atender os preceitos legais que definem como obrigatórias estas práticas para garantir o certificado de filantropia, o discurso dos gestores institucionais elege como prioridade a interação com o mercado (BRISOLLA et al, 1997). Esta priorização das relações com o ambiente externo não é afirmada em detrimento ao ensino, e sim, pela complementaridade, de forma e conteúdo, que as atividades decorrentes da interação podem proporcionar. Os gestores institucionais possuem uma clara percepção de que a atividade de ensino já está devidamente internalizada, não havendo necessidade de maiores ajustes, diferentemente da pesquisa e extensão que tradicionalmente estavam à margem das políticas institucionais e nunca chegaram a ter representatividade significativa dentro das ações e receitas da universidade (HOFSTEDE et al, 1990; CARTER, 1998).

A respectiva representatividade era mínima principalmente em receitas financeiras devido ao fato de que universidade financiava com recursos próprios a realização de pesquisas, tanto de horas de professores/ pesquisadores como do material de custeio, onerando os custos operacionais da universidade. Com as alterações ambientais, caracterizadas principalmente pela entrada de novos concorrentes na região, com estruturas institucionais menos onerosas e, desta forma, podendo praticar valores mais baixos, a sustentação do financiamento interno das pesquisas se tornou inviável. O próprio perfil das pesquisas realizadas com o financiamento interno era, na sua maioria, apenas de cunho teórico e não aplicado, agregando valor à produção científica do pesquisador, de pontuação da 


\section{A INFLUÊNCIA DA CULTURA ORGANIZACIONAL UNIVERSITÁRIA SOBRE O PROCESSO DE TRANSFERÉNCIA TECNOLÓGICA \\ DOI: http://dx.doi.org/10.5007/1983-4535.2014v7n3p247}

instituição junto aos órgãos governamentais, mas de pouco benefício para a comunidade de entorno, principalmente da comunidade empresarial (SOUZA, 1978; MACHADO, 2005).

Com o advento da nova realidade, de menor ingresso de alunos, menor receita operacional, a prática de financiamento interno das pesquisas foi a primeira a ser reduzida, em primeiro momento, e eliminada, em momento posterior. A instituição, seguindo modelos de outras universidades, tanto privadas como públicas, incluiu no eixo central das mudanças estratégicas estruturais, a captação de recursos externos, tanto de origem privada, como pública, para o financiamento das pesquisas acadêmicas, que a partir desse momento deveriam atender não apenas as necessidades do pesquisador e da ciência, como também as necessidades da comunidade, mais especificamente, dos financiadores dos respectivos projetos de pesquisa (ETZKOWITZ, 1998; BRISOLLA, 1997).

Esta retórica ficou clara para o corpo gestor da universidade, mas encontrou resistência junto ao corpo docente, evidenciado uma ruptura velada do consenso institucional, de produção científica. O domínio dos ritos administrativos internos de submissão de propostas de financiamento de pesquisas internas, de cunho eminentemente teórico, não encontrava o reconhecimento junto às fontes financiadoras externas e a produção de pesquisas praticamente deixou de existir (BERTHON et al, 2001; HUMPRHREYS \& BROWN, 2002).

As entrevistas em profundidade realizadas com os doze docentes dos seis cursos tecnológicos revelaram, no entanto, que as percepções do processo de interação da U-E e da necessidade de desenvolver novos tipos de propostas de pesquisa, que atendessem tanto as necessidades de agregar novos conhecimentos científicos, como as de sua aplicação para a geração de novos produtos ou de aperfeiçoamento de processos industriais, apresentaram significativas variações. Estas variações eram de menor intensidade dentro do curso e mais intensas entre os cursos e, principalmente, apresentavam alto grau de heterogeneidade de conteúdo do discurso entre os docentes dos seis cursos em relação ao discurso dos gestores institucionais (BRISOLLA et al, 1997).

Foi verificado, também, que o teor das entrevistas dos coordenadores dos seis cursos tecnológicos apresentava, em muitos pontos, maior aderência à retórica dos gestores institucionais do que à percepção dos docentes do curso pelo qual o coordenador era responsável, demonstrando que, ao menos no discurso, o coordenador se sentia obrigado a defender os interesses institucionais, contidos e representados no novo plano de ação da universidade. Apesar dos pontos de convergência em maioria dos pontos abordados em todas 


\section{A INFLUÊNCIA DA CULTURA ORGANIZACIONAL UNIVERSITÁRIA SOBRE O PROCESSO DE TRANSFERÉNCIA TECNOLÓGICA \\ DOI: http://dx.doi.org/10.5007/1983-4535.2014v7n3p247}

as entrevistas, verificou-se maior coesão no discurso do coordenador com os docentes em pontos considerados críticos no processo de interação U-E, como a infra-estrutura de apoio e pagamento de horas de pesquisa para a preparação de propostas de pesquisa para os órgãos de fomento como FINEP e CNPQ, e para a negociação de propostas de transferência tecnológica junto às empresas da região. Nestes pontos, onde a responsabilidade do processo impactava nas metas individuais do próprio coordenador, o conteúdo do discurso apresentava maior divergência do teor da entrevistas com os gestores universitários (PIRES \& MACEDO, 2006; GUNASEKARA, 2006).

Em se tratando das percepções quanto ao processo de encaminhamento de propostas de projeto junto aos órgãos de fomento e junto às empresas, o conteúdo das vinte e quatro entrevistas apresentava alto grau de alinhamento, com exceção de apenas um docente, oriundo de uma universidade pública, com denso histórico de projetos de pesquisa aprovados e executados com financiamento público e de empresas. Os demais entrevistados, independentemente da posição hierárquica ocupada dentro da instituição, definiam o acesso aos recursos da FINEP e CNPQ como burocrático e os critérios de aprovação destes órgãos como pouco transparentes.

No entanto, este consenso apresentou ruptura em caso de negociação de propostas de transferência tecnológica junto a empresas da região. Enquanto que para os gestores universitários era considerada mais fácil, devido ao menor nível de exigências burocráticas tanto junto a empresas como junto a instâncias internas de aprovação de propostas de pesquisas externas, tanto os docentes como os coordenadores dos cursos tecnológicos apontaram vários fatores dificultadores do processo como: (i) a necessidade de investimento inicial de horas e recursos financeiros por parte do docente para a atividade de prospecção e elaboração da proposta junto à empresa; (ii) diferenças no ritmo e nas prioridades da empresa e da universidade quanto a aplicação dos resultados da pesquisa e da transferência do conhecimento - enquanto que a universidade prioriza o grau de certeza dos resultados da pesquisa, em detrimento do tempo de pesquisa, a empresa prefere o contrário, para garantir a sua posição de inovador no mercado e ser o primeiro a lançar o produto; (iii) a falta de consenso quanto a remuneração de horas de pesquisa entre o docente e a empresa; (iv) considerável burocracia interna para a utilização dos ativos e demais recursos da universidade, como veículos e laboratórios, para a realização de projetos de pesquisa; (v) a demora e burocracia interna para a efetivação do contrato entre a universidade e a empresa; (vi) a falta 


\section{A INFLUÊNCIA DA CULTURA ORGANIZACIONAL UNIVERSITÁRIA SOBRE O PROCESSO DE TRANSFERÉNCIA TECNOLÓGICA \\ DOI: http://dx.doi.org/10.5007/1983-4535.2014v7n3p247}

de colaboração de instâncias hierárquicas responsáveis pela aprovação das propostas de pesquisa - pela necessidade institucional de que estas agregassem também à produção científica - comprometendo o desenho das propostas já aprovadas pelas empresas, frustrando as expectativas tanto das empresas como dos pesquisadores; (vii) a demora e formas de repasse dos recursos financeiros decorrentes do contrato com as empresas para os pesquisadores, em virtude de centralização e gerenciamento destes recursos em outros departamentos da universidade; (viiii) remuneração das horas de pesquisa em folha de pagamento, na modalidade de horas aula, com valor reduzido devido a encargos sociais e ainda sofrendo a incidência de tributação sobre os rendimentos de pessoa física, desestimulando o pesquisador do projeto.

Os docentes entrevistados, especialmente os com mais tempo de vínculo institucional e maior influência no corpo docente do curso, destacavam que o processo de interação com empresas da região era mais intenso quando os cursos tecnológicos possuíam maior autonomia dentro da instituição para atuar, em virtude de uma agência própria de gestão e tecnologia que foi desativada no processo de reestruturação institucional, sendo suas atribuições transferidas para o departamento de assessoria à diretoria, que passou a centralizar a gestão de projetos de toda a universidade (ETZKOWITZ, 1998; GUNASEKARA, 2006).

As críticas que os docentes e também os coordenadores apresentaram ao novo modelo de gestão das relações com o mercado referiam-se à composição do quadro de profissionais do departamento responsável pela coordenação das atividades que não possuía o conhecimento técnico dos projetos tecnológicos, para poderem negociar com as instituições de apoio e com as empresas, nem a experiência corporativa, empresarial, que facultasse o entendimento da "linguagem" dos empresários e executivos das empresas cliente. Esta falta de empatia comprometia, na percepção de docentes, o processo de interação U-E (BOLON \& BOLON, 1994; HUMPRHREYS \& BROWN, 2002).

No tocante ao foco institucional que passou a priorizar as relações com a comunidade, no caso dos cursos tecnológicos a comunidade empresarial, constatou-se clara divergência entre a percepção do grupo docente e dos gestores institucionais, com os coordenadores situados entre os dois extremos. Enquanto para a maioria dos docentes o ensino permanecia a prioridade institucional, com a pesquisa e extensão configurando um extra, mas sem maior validação institucional, para os gestores estava clara a determinação da alta gestão de inserção da cultura de pesquisa e de atividades de extensão nos cursos tecnológicos. 


\section{A INFLUÊNCIA DA CULTURA ORGANIZACIONAL UNIVERSITÁRIA SOBRE O PROCESSO DE TRANSFERÉNCIA TECNOLÓGICA \\ DOI: http://dx.doi.org/10.5007/1983-4535.2014v7n3p247}

Cada um dos grupos evidenciava o teor de sua argumentação em documentos internos, como normas, procedimentos internos, planos de trabalho, planos de ação, planos de atividade docente, comunicados internos, atas de reuniões, entre outros, nos quais se respaldavam para evidenciar que a instituição priorizava o ensino (grupo de docentes) e priorizava também as atividades de pesquisa e extensão, por considerar a atividade de ensino já consolidada (gestores institucionais). Entre o grupo de coordenadores verificou-se que maioria estava ciente das novas orientações da diretoria mas permaneciam focados em ensino, pois as metas e objetivos com os quais tinham se comprometido, tratavam basicamente de ensino e denotavam indicadores como redução de evasão dos alunos, acompanhamento de professores, realização de reuniões, elaboração de relatórios e atas, além de cumprimento de metas de produção científica (BERTHON et al, 2001; TERRA \& ETZKOWITZ, 2007).

Durante as entrevistas, os grupos de docentes, inclusive de coordenadores, referiam-se à administração institucional como "o pessoal lá de cima", deixando evidente que existe um distanciamento tanto discursivo como físico entre a gestão e o corpo docente, ao menos, dos cursos tecnológicos (SOUZA, 1978; MACHADO, 2005). Esta verbalização foi reforçada por observações como "eles lá e nós aqui" onde o grupo de docentes deixava claro que existiam dentro da universidade "mundos" diferentes e regidos com regras próprias. Todas as entrevistas retratavam a importância da comunicação institucional no processo de compartilhamento de políticas e valores institucionais, ainda mais no período de revisão de estratégias e formas de atuação (ALBERT, 2000).

Alguns meses antes da realização das entrevistas a instituição deu início ao processo de reestruturação do quadro docente, com desligamento de vários profissionais com muitos anos de vínculo com a universidade e contratação de novos membros, com o perfil mais próximo do que a instituição percebia como ideal para enfrentar os desafios do mercado de ensino. Tratava-se de profissionais com vasta experiência em pesquisa e que eram contratados com o objetivo de compartilhar o seu know-how com os demais docentes da instituição.

No entanto os novos profissionais, ao "descer" aos seus locais de trabalho, após o período de integração, ou aderiam ao modus operandi do grupo, ou ficavam isolados, como era o caso de um dos docentes entrevistados, que integrou o corpo docente, mas não o grupo de docentes do curso, por possuir perfil de pesquisador, com projetos CNPQ e FINEP em andamento e interesse em se dedicar ás suas pesquisas. Ao não encontrar o respaldo entre os 


\section{A INFLUÊNCIA DA CULTURA ORGANIZACIONAL UNIVERSITÁRIA SOBRE O PROCESSO DE TRANSFERÉNCIA TECNOLÓGICA \\ DOI: http://dx.doi.org/10.5007/1983-4535.2014v7n3p247}

colegas, permaneceu com vínculos e contatos externos à instituição, tanto junto a empresas, como dentro das instituições de fomento (BLANCHARD, 1982; MAEL \& ASFORTH, 1992).

Finalmente, em todas as entrevistas realizadas ficou evidente a importância do processo de comunicação institucional para o compartilhamento dos valores, significados e direcionamento, principalmente quando a organização passa por mudanças estruturais, que impactam diretamente sobre a realidade organizacional construída gradativamente ao longo dos anos com base em valores como fidelidade, estabilidade, garantia de direitos, carreira, proteção e segurança, os quais não resistiram à influência do meio. Foi ressaltada a necessidade vital de diálogo institucional como condição sine qua non para a reconstrução do clima organizacional favorável à realização de acordos implícitos entre a alta gestão e o corpo docente (GUNASEKARA, 2006).

\section{CONSIDERAÇÕES FINAIS}

O debate da importância da interação U-E para o desenvolvimento econômico e social não é recente. No entanto a atualidade do tema é mantida devido a falta de consenso no que se refere ao modelo ideal para realizar a efetiva interação entre a academia e indústria. Vários autores e pesquisadores apontaram uma série de motivos e fatores que possivelmente dificultam a interação U-E, mas poucos estudos se detiveram sobre a dimensão da cultura organizacional universitária como a variável que possa ocupar o papel de relevância no processo e, por conseguinte, facilitar ou dificultar a respectiva interação. A presente pesquisa foi conduzida com o objetivo de colaborar neste sentido e procurar contribuir para o debate em tela. Conduzida por meio do método de estudo de caso e utilizando a técnica de entrevistas em profundidade, vinte e quatro profissionais vinculados a uma universidade privada de grande porte confirmaram as vertentes teóricas que retratam o ambiente universitário como um locus de múltiplas realidades percebidas por atores que dela participam influenciando o processo de interação U-E da referida instituição.

Apoiando-se no conjunto de valores que até então eram cultivados e referenciados na instituição, como a flagrante priorização da excelência do ensino, estabilidade, garantia de direitos adquiridos, benefícios, carreira e proteção institucional da competição acirrada e agressividade do mundo externo, o corpo docente foi colocado de frente a uma nova realidade organizacional, reconstruída pela alta gestão, em virtude das pressões que a instituição vinha recebendo do ambiente externo. Alguns sinais destas pressões já eram percebidos 


\section{A INFLUÊNCIA DA CULTURA ORGANIZACIONAL UNIVERSITÁRIA SOBRE O PROCESSO DE

internamente, como a redução no número de alunos, programas de desligamento voluntário e reestruturação funcional e organizacional.

Com o objetivo de reduzir os custos e ajustar a equipe ao novo patamar de receitas operacionais, a instituição adotou um novo modelo de gestão, com eliminação de níveis hierárquicos e de áreas e departamentos. A centralização das atividades de coordenação do processo de interação U-E na área de gestão, sob a tutela de profissionais de gestão e sem conhecimentos prévios nem na área de tecnologia e nem em negociação com empresas e órgãos de fomento, impactou de forma negativa no ambiente organizacional.

O conjunto de novos valores institucionais, derivados do reposicionamento estratégico da universidade recebeu resistência velada por parte do corpo docente, evidenciada pelo emprego da percepção seletiva de normas, procedimentos e determinações. O processo de comunicação interna não foi suficientemente capaz de superar a ruptura organizacional interna, com a gestão de um lado e o corpo docente do outro e os esforços institucionais empreendidos no sentido de fomentar a interação U-E, com o objetivo de (i) aumentar o ingresso de receitas adicionais via projetos de pesquisa aplicada e (ii) manter e aumentar a produção acadêmica, foram frustrados pela condução inadequada do processo de compartilhamento de novos valores institucionais, baseados numa nova realidade externa.

Identificou-se que o grupo de docentes dos cursos tecnológicos mantém uma idiocultura particular, com valores, objetivos e foco próprios, apoiados em experiências históricas compartilhadas, tanto de caráter individual como do grupo, interpretados à luz de percepções de docentes mais antigos e detentores da memória organizacional deste grupo. Esta cultura particular dos docentes dos cursos tecnológicos é, por sua vez, composta por culturas de grupos menores, de cada um dos seis cursos tecnológicos, devido a características específicas e próprias, de docentes que fazem parte daquele curso em questão, diferindo em experiências individuais compartilhadas, visões da realidade organizacional e do espaço ocupado dentro da organização, tanto pelo curso, como por docentes, como grupo, em relação à gestão.

Para reforçar a estrutura do grupo e manter a visão cultural compartilhada, o grupo de docentes encontra apoio em símbolos de afirmação cultural e narrativas construídas com base em acontecimentos que mais marcaram (de forma positiva ou negativa - na percepção do grupo e dos profissionais formadores de opinião) o grupo, como os casos de sucesso, a autonomia conquistada quando da existência do NAI e depois perdida, com a centralização do 


\section{A INFLUÊNCIA DA CULTURA ORGANIZACIONAL UNIVERSITÁRIA SOBRE O PROCESSO DE TRANSFERÉNCIA TECNOLÓGICA \\ DOI: http://dx.doi.org/10.5007/1983-4535.2014v7n3p247}

processo de interação com o mercado para o departamento localizado junto à administração institucional. A interpretação do conjunto de narrativas históricas, símbolos e experiências individuais e coletivas, resulta na percepção da nova realidade institucional e de características que esta nova configuração apresenta em termos de oportunidades e ameaças para o grupo, moldando e influenciando as ações, tanto individuais, como grupais.

A presente pesquisa procurou identificar e dimensionar as variáveis que influenciaram o processo de interação U-E, oriundas de fatores culturais intrínsecos da organização pesquisa, visando demonstrar os vínculos que existem entre a cultura organizacional e os propósitos institucionais, seus processos formais e desta forma demonstrar a importância da gestão da cultura organizacional. Esta preocupação dos pesquisadores se deve à constatação de que a maioria dos estudos vincula o desempenho aquém do esperado, na interação U-E, à diferença cultural entre os ambientes acadêmico e empresarial, sendo que em universos organizacionais mais complexos, como é o caso do ambiente universitário, a cultura é um fenômeno multidimensional e composto por idioculturas ou sub-culturas organizacionais, que por si só têm o poder de se contrapor aos valores institucionais.

Os autores da presente pesquisa procuraram retratar de como a cultura organizacional, particular e específica de grupo de docentes dos cursos tecnológicos de uma universidade pode exercer a influência sobre o processo de interação U-E, mas entendem que o tema mercê mais pesquisas, sobretudo de natureza quantitativa, com o objetivo de mensurar e dimensionar os aspectos culturais intervenientes.

\section{REFERÊNCIAS}

ALBERT, Stuart; ASHFORTH, Blake E; DUTTON, Jane E. Organizational identity and identification: charting new waters and building new bridges. Academy of Management Review, v. 25, n. 1, p. 13-17, 2000.

BERTHON, Pierre; PITT, Leyland F; EWING, Michael T. Corollaries of the collective: The influence of organizational culture and memory development on perceived decision-making context. Journal of Academy of Marketing Science. v. 29, n.2; p. 135-150, 2001.

BLANCHARD, K H.; HERSEY, P.; JOHNSON, D. Management of Organizational Behavior: Utilizing Human Resources. Englewood Cliffs: Prentice Hall, 1982.

BOLON, Douglas S, BOLON, Donald S. A reconceptualization and analysis of organizational culture. Journal of Managerial Psychology. Bradford: v.9, n. 5; p. 22-27, 1994. 


\section{A INFLUÊNCIA DA CULTURA ORGANIZACIONAL UNIVERSITÁRIA SOBRE O PROCESSO DE TRANSFERÉNCIA TECNOLÓGICA \\ DOI: http://dx.doi.org/10.5007/1983-4535.2014v7n3p247}

BRISOLLA, Sandra; CORDER, Solange; GOMES, Erasmo; MELLO, Débora. As relações universidade-empresa-governo: Um estudo sobre a Universidade Estadual de Campinas (UNICAMP). Educação \& Sociedade.v.18, n.61, p.187-209, 1997

CARTER, Suzanne M. Corporate Responses to Changes in Reputation. Corporate Reputation Review,v 1, n 3, p. 250-270, 1998.

CHIN-LOY, Claudette, HUIZENGA, Wayne. Assessing the influence of organizational culture on knowledge management success. Tese de Doutorado da School of Business and Entrepreneurship from Nova Southeastern University, 2003.

ETZKOWITZ, Henry. The norms of entrepreneurial science: cognitive effects of the new university-industry linkages. Research Policy. v. 27, n.8-9, p.823-833, 1998.

GUNASEKARA, Chrys. Reframing the Role of Universities in the Development of Regional Innovation Systems. The Journal of Technology Transfer, v 31, n.1, p. 101-113, 2006. HOFSTEDE, Geert; NEUIJEN, Bram; OHAYV, Denise Daval; SANDERS, Geert. Measuring Organizational Cultures: A Qualitative and Quantitative Study Across Twenty Cases. Administrative Science Quarterly, v.35, n.2; p. 286-316, 1990.

HUMPRHREYS, M.; BROWN, A. Narratives of organizational identity and identification: a case study of hegemony and resistance. Organizational Studies, v. 23, n. 3, p. 421-447, 2002.

LEYDESDORFF, Loet; ETZKOWITZ, Henry. Report of the Fourth Triple Helix Conference in Copenhagen (6-9 November 2002). Disponível em: www.leydesdorff.net/th4/spp.htm. Acessado em 20/04/2007.

MACHADO, Hilka Vier. Identidade Organizacional: Um Estudo de Caso no Contexto da Cultura Brasileira, UEM, RAE-eletrônica, v. 4, n. 1, Art. 12, jan./jul. 2005.

MAEL, F.; ASFORTH, B. Alumni and their alma mater: a partial test of the reformulated model of organizational identification. Journal of Organizational Behavior, v. 13, p. 103-123, 1992.

MAGEE, Kimberly Clauss. The Impact of Organizational Culture on the Implementation of Performance Management. Tese de Doutorado da Georgia State University, 2002.

MOTTA, F. C.; CALDAS, M. Cultura organizacional e cultura brasileira. São Paulo: Atlas, 1997.

OUGHTON, Christine; LANDABASO, Mikel; MORGAN, Kevin. The Regional Innovation Paradox: Innovation Policy and Industrial Policy. Journal of Technology Transfer; 27, 1; pg. 97-110, 2002.

PIRES, José Calixto de Souza; MACEDO, Kátia Barbosa. Cultura organizacional em organizações públicas no Brasil. RAP-Revista de Administração Pública, Rio de Janeiro v.40, n.1, p.81-105, Jan./Fev. 2006. 


\section{A INFLUÊNCIA DA CULTURA ORGANIZACIONAL UNIVERSITÁRIA SOBRE O PROCESSO DE TRANSFERÊNCIA TECNOLÓGICA \\ DOI: http://dx.doi.org/10.5007/1983-4535.2014v7n3p247}

PLYMIRE, Jerry. The Depth Psychological Implications of Ogranizational Culture. Tese de Doutorado da Pacifica Graduate Institute. 1993.

SABATO, J. A.; BOTANA, N. La ciencia y la tecnología en el desarrollo futuro de América Latina in: SABATO, J. A. El pensamiento latinoamericano en la problemática cienciatecnología-desarrollo-dependencia, Buenos Aires: Paidos, 1975. p. 132-143.

SCHEIN, E. H. Organization Culture and Leadership. San Francisco: Jossey Bass, 1988.

SOUZA, Edela L. P. Clima e Cultura organizacional: como se manifestam e como se manejam. São Paulo: Edgard Blücher, 1978.

SUGATO, Lahiry. Building commitment through organizational culture. Training \& Development; v.48, 4; p. 50-52, 1994.

TERRA, Branca; ETZKOWITZ, Henry. A Universidade Empreendedora e a Sociedade da Nova Era. Disponível em: www.competenet.org.br/evento/branca.pdf. Acessado em 15/04/2007. 